\title{
Homens e masculinidades e o novo coronavírus: compartilhando questões de gênero na primeira fase da pandemia
}

\author{
Men, masculinity and the new coronavirus: \\ sharing gender issues in the first phase of the pandemic
}

Benedito Medrado (https://orcid.org/0000-0002-1085-5024) ${ }^{1}$

Jorge Lyra (https://orcid.org/0000-0002-5381-2059) ${ }^{1}$

Marcos Nascimento (https://orcid.org/0000-0002-3363-4232) ${ }^{2}$

Adriano Beiras (https://orcid.org/0000-0002-1388-9326) ${ }^{3}$

Áurea Christina de Paula Corrêa (https://orcid.org/0000-0003-2091-6879) ${ }^{4}$

Eric Campos Alvarenga (https://orcid.org/0000-0002-1803-2356) ${ }^{5}$

Maria Lucia Chaves Lima (https://orcid.org/0000-0003-3062-2399) ${ }^{5}$

\footnotetext{
${ }^{1}$ Departamento de Psicologia, Centro de Filosofia e Ciências Humanas, Universidade Federal de Pernambuco. R. Academico Hélio Ramos s/n, Cidade Universitária. 50740-530 Recife PE Brasil.beneditomedrado@ gmail.com

${ }^{2}$ Instituto Nacional de Saúde da Mulher da Criança e do Adolescente Fernandes Figueira, Fiocruz. Rio de Janeiro RJ Brasil.

${ }^{3}$ Departamento de Psicologia, Centro de Filosofia e Ciências Humanas, Universidade Federal de Santa Catarina. Florianópolis SC Brasil.

${ }^{4}$ Faculdade de Enfermagem, Universidade Federal de Mato Grosso. Cuiabá MT Brasil.

${ }^{5}$ Faculdade de Psicologia, Universidade Federal do Pará. Belém PA Brasil.
}

\begin{abstract}
This article presents reflections on masculinity and the social construction of genderbased on the global phenomenon of the new coronavirus pandemic - produced by researchers who are part of the national research team on comprehensive health care policy for men in Brazil. From a gender-based standpoint, the article contends that it is necessary to note that cis heteronormative male socialization is guided by three core issues: 1) the submission to practices of care of self and others; 2 ) the rejection of preventive health practices, due to a distorted matrix of risk perception (and a certain sense of "invulnerability"); 3) the domestic dynamics marked by postures of command, order, and honor. These dimensions of everyday life were profoundly upset in this first phase of the epidemic, in which confinement became the most recommended alternative. These issues are configured as recurring (though not recent) repertoires that glorify the central model of a male order that needs to become an object of reflection, insofar as they endanger the health of men and women and, more broadly, of the status quo of the accepted tenets of domestic and social order.
\end{abstract}

Key words Men, Masculinity, Health, Covid
Resumo Este artigo opinião apresenta reflexões sobre masculinidades e construções de gênero - a partir do fenômeno global da pandemia do novo coronavírus -, produzidas por pesquisadores/as que integram a equipe nacional de uma pesquisa sobre política de atenção integral aos homens na saúde, no Brasil. A partir de leituras baseadas em gênero, o artigo argumenta que é preciso atentarmos que a socialização masculina cisheteronormativa se orienta a partir de três eixos: 1) a abjeção às práticas de cuidado de si e dos outros; 2) a rejeição às práticas preventivas em saúde, dada uma distorcida matriz de percepção de risco (e certo sentimento de "invulnerabilidade"); 3) a dinâmica doméstica marcada por posições de comando, ordenamento e honra. Essas dimensões da vida cotidiana foram profundamente provocadas nesta primeira fase da epidemia, em que o confinamento se tornou a alterativa mais recomendável. Esses eixos se configuram como repertórios recorrentes (embora não recentes) que reificam o modelo central de uma ordem masculina que precisa se tornar objeto de reflexão, na medida em que colocam em risco a saúde de homens e mulheres e mais amplamente dos pactos civilizatórios e da ordem social.

Palavras-chave Homens, Masculinidades, Saúde, Covid 


\section{Introdução}

Vivemos atualmente um dos momentos mais difíceis da história mundial recente, a pandemia do SARS-CoV-2, conhecido como "novo coronavírus". Esse preocupante cenário tem produzido alterações profundas em nossas condições de vida e no modo como nos relacionamos. Na mesma medida, por um lado, passamos a perceber, de modo mais nítido, problemas crônicos que antes, em condições de suposta "normalidade", pareciam não existir e, por outro lado, nenhum conhecimento acumulado até o momento tem nos dado segurança sobre possibilidades efetivas e estratégias conclusivas sobre imunização, tratamento e cura para a COVID-19. No cenário global, o Brasil é o segundo país com maior número de pessoas contaminadas e de óbitos, atrás apenas dos Estados Unidos'.

As primeiras publicações no campo da saúde coletiva já apontam para uma sobremorbidade masculina. Em um artigo publicado em fevereiro de 2020, por pesquisadores/as chineses/as, faz-se uma breve análise sobre os casos confirmados, que deram entrada entre $1^{\circ}$ e 20 de janeiro de 2020 em um hospital de Wuhan, o provável epicentro do surto. De 99 pessoas pesquisadas, 67 eram do sexo masculino $(68 \%)^{2}$.

Esses/as pesquisadores/as se adiantam em argumentar que "a susceptibilidade reduzida das mulheres às infecções virais pode ser atribuída à proteção do cromossomo $\mathrm{X}$ e dos hormônios sexuais, que desempenhariam um papel importante na imunidade inata e adaptativa"'. Essa conclusão precipitada teve por base apenas outro artigo, publicado em 2019 (sobre dimorfismo sexual na imunidade inata), ignorando a histórica controvérsia inato versus adquirido, na Epidemiologia, e uma vasta literatura sobre determinantes sociais em saúde, particularmente relacionados à dimensão cultural de gênero.

Neste campo profícuo de incertezas, consideramos precoce qualquer leitura que possa ser feita com vista à produção de respostas, o grupo de pesquisadores/as que assina o presente artigo resolveu compartilhar, por meio deste breve ensaio, algumas questões que têm alimentado nossos debates, hoje ainda mais pungentes, sobre atenção integral à saúde do homem, a partir de uma perspectiva feminista de gênero, e que podem contribuir para dar visibilidade a outras questões importantes na conjuntura atual.

Tais questões têm por base leituras baseadas em gênero, que temos feito no desenvolvimento de uma pesquisa, que teve início em 2018, e envolveu um conjunto de pesquisadores/as vinculados/as a universidades e centros de pesquisa das cinco regiões do país (UFPE, UFPA, UFSC, UFMT e Fiocruz). Nesta pesquisa, operamos com estratégias metodológicas diversas e dialogamos com diferentes interlocutores/as - entre gestores, profissionais de saúde, usuários e potenciais usuários dos serviços de atenção primária em saúde. Nosso objetivo é produzir memória sobre a primeira década de implementação da política e dialogar sobre conteúdo, atores, contextos e processos que conformam os primeiros passos dessa política ${ }^{4}$. Cabe ressaltar que o Brasil é um dos poucos países que possui uma política de saúde específica voltada para a população masculina, seguido apenas pela Irlanda, Austrália e Irã ${ }^{5}$.

Sendo um país de dimensão continental, com desigualdades sociais entre suas diferentes regiões e com distintas perspectivas de gestão política e sanitária à pandemia, é preciso reconhecer a multiplicidade de facetas que esta assume no cenário nacional. Desse modo, consideramos que nossas reflexões são ainda iniciais, atendo nossas leituras ao período que compreende o primeiro semestre de 2020, no qual temos experimentado, com maior ou menor rigor, a difícil e necessária medida de prevenção baseada no isolamento social, seguindo orientações de entidades e profissionais, baseados em protocolos da Organização Mundial de Saúde. Além disso, parece-nos importante destacar a necessidade de pensar o processo saúde-doença-cuidar a partir de uma perspectiva de gênero ${ }^{6}$, aqui compreendida como abordagem analítica que nos permite estudar regulações e determinadas ordens sociais e produção de sentidos inclusive sobre cuidado e a saúde; e, em particular, no contexto de pandemias ${ }^{7}$.

Reconhecemos que no período inicial da pandemia, a intensa busca pela aquisição de respiradores e de equipamentos de proteção individuais para trabalhadores e trabalhadoras da saúde, assim como a criação de mais leitos de UTI em hospitais foi de extrema relevância. Da mesma forma que os esforços coordenados por entidades brasileiras em colaboração com centros de pesquisa internacionais na descoberta de uma possível vacina. Contudo, é preciso trilhar por outros caminhos, de modo adicional e não substitutivo. O fortalecimento da Estratégia de Saúde da Família, por exemplo, pode significar um aumento em práticas de prevenção e promoção à saúde por meio de sua proximidade com as pessoas em seus territórios, tendo agentes comunitários de saúde como peça chave para levar informações às famílias. 
Neste cenário de confinamento, é relevante atentar, por exemplo, que algumas pessoas já moraram sós e passaram a viver sós. Outras passaram a conviver 24 horas com pessoas com quem só se encontravam no café da manhã, à noite e nos fins de semana. Outras estão sendo obrigadas a conviver com quem os conflitos e a violência já eram a dinâmica cotidiana. Com isso, invariavelmente, há um potencial incremento de agravos à saúde mental e preocupações, particularmente dos movimentos sociais feministas, em relação à violência doméstica e familiar, baseada em gênero e sexualidade. Em uma sociedade machista e patriarcal como a brasileira, em que foi necessária a institucionalização de uma lei para coibir práticas de violência doméstica e familiar, baseada em gênero, definitivamente não é exagero pensar que as mulheres, crianças, adolescentes, idosos e LGBTQI+ estão, em período de confinamento, ainda mais vulneráveis, particularmente em contexto doméstico e familiar ${ }^{8}$.

Na mesma medida, é preciso atentarmos que a socialização masculina cisheteronormativa se orienta a partir de três eixos: 1) a abjeção às práticas de cuidado de si e dos outros; 2 ) a rejeição às práticas preventivas em saúde, dada uma distorcida matriz de percepção de risco (e certo sentimento de "invulnerabilidade"); 3 ) a dinâmica doméstica marcada por posições de comando, ordenamento e honra. Essas dimensões da vida cotidiana foram profundamente provocadas nesta primeira fase da epidemia, em que o confinamento se tornou a alterativa mais recomendável ${ }^{9}$.

O contexto econômico de uma pandemia global também deve ser considerado neste cenário de intensificação da violência doméstica e familiar, a partir de leituras mais estruturais. Um abalo nas taxas de desemprego já começa a ser sentido tanto no Brasil ${ }^{10}$ como em outros países ${ }^{11}$. Considerando o trabalho enquanto um ponto fundamental para a vivência das masculinidades de diversos homens, a experiência de não estar empregado e a perda do "lugar" de "provedor da família" pode ter relação estreita com o aumento das agressões no ambiente doméstico e fora dele.

O confinamento também intensificou os afazeres domésticos que, na ordem econômica do cuidado (seja formal ou informal), são tradicionalmente delegados às mulheres, e a pessoas que, por sua condição de classe social, não têm alternativas. Considerando que a socialização dos homens, na atualidade, ainda os desresponsabiliza e os descapacita para práticas de cuidado, é importante reconhecer a sobrecarga de trabalho doméstico para as mulheres, em diferentes idades, em tempos de confinamento.
Na mesma medida, não podemos ignorar que a socialização masculina, notadamente marcada pela valorização da honra e da virilidade ${ }^{9,12}$, produz-se a partir de um modelo cultural ideal que, mesmo não sendo atingível por praticamente homem algum, exerce sobre os homens e sobre as mulheres um efeito de regulação e controle ${ }^{13}$. Neste modelo ideal-branco, cisheteronormativo, patriarcal e colonial -, o cuidado é uma prática considerada feminina, sendo o risco considerado pela via do enfrentamento e não da prevenção. A sensação de "invulnerabilidade masculina" é um efeito possível e esperado dessa economia de gênero. Entretanto, é preciso considerar a dialética de gênero que se configura nessa economia de gênero pois, por um lado, tal invulnerabilidade e aversão ao cuidado não necessariamente performa a prática de todo e qualquer homem, e, por outro, essa posição de sujeito pode resultar em consequências complexas como, por exemplo a morte de um em cada cinco homens antes de completar 50 anos de idade, a grande maioria por causas externas, e uma expectativa de vida de 5,8 anos a menos em comparação com as mulheres na região das Américas ${ }^{14}$. Ou ainda, o fato de que os níveis de mortalidade masculina decorrente da pandemia sejam superiores à feminina na maioria dos países analisados ${ }^{15}$.

Portanto, novamente é consequente pensar que homens, socializados em nossa cultura, tornem-se potenciais vetores de infecção, não porque eles "são como são", mas porque foram socializados e estimulados a ocuparem os espaços públicos, sem restrições e posição de sujeito, em uma sociedade que valoriza e premia certos atributos associados a modos de subjetivação masculina, dificultando algumas das principais práticas para evitar à disseminação da COVID-19, que são o isolamento social, o uso de máscaras e a higienização das mãos.

Gostaríamos de ressaltar, porém, que pensar sobre homens e masculinidades, em uma perspectiva feminista de gênero vai além de leituras sobre modos de socialização e de subjetivação masculina. Afinal, masculinidade e feminilidade não são associadas, respectivamente, a homens e mulheres cisgêneros ${ }^{16}$. São, como adverte Miguel Vale de Almeida ${ }^{17}$, metáforas de poder e de capacidade de ação e, como tal, podem ser acessadas por homens e mulheres, independente de orientação sexual e identidade de gênero, embora com efeitos particularmente diferentes. E, na mesma medida podem se expressar em materialidades diversas e, inclusive, em práticas discursivas.

Nestes primeiros meses da pandemia, por exemplo, temos visto acirrarem-se controvér- 
sias em torno da estratégia do confinamento, em meio a disputas políticas, de ordem eleitoral e a recorrentes práticas discursivas machistas e patriarcais, que colocam em relevo a ordem produtiva em detrimento da ética do cuidado e da vida $^{18}$. Fica-nos o seguinte questionamento: é possível transformar os sentidos dados ao cuidado ou "desgenerificá-lo" em prol de uma transformação e maior efetividades das estratégias coletivas de redução do contágio da pandemia?

Não por acaso, como noticiado pela imprensa brasileira, que o presidente Jair Bolsonaro disse, em pronunciamento público, que é preciso enfrentar o problema "como homem, pô, não como moleque", em um passeio pelo comércio de Brasília e cidades vizinhas, na manhã do dia 29/03/2020, "contrariando mais uma vez o [então] ministro da Saúde, Luiz Henrique Mandetta, e autoridades médicas de todo o mundo que defendem o isolamento social contra o novo coronavírus" ${ }^{\prime 19}$. Na mesma medida, vemos expressões desse ordenamento machista, em seu pronunciamento em rede nacional, ao afirmar que "no meu caso particular, pelo meu histórico de atleta, caso fosse contaminado pelo vírus, não precisaria me preocupar. Nada sentiria ou seria, quando muito, acometido de uma gripezinha ou resfriadinho"20.

\section{Colaboradores}

B Medrado, J Lyra, M Nascimento, A Beiras, ACP Corrêa, EC Alvarenga e MLC Lima participaram da produção das informações, revisão bibliográfica e construção dos argumentos que deram sustentação à redação e revisão do artigo. $\mathrm{B}$ Medrado, na condição de primeiro autor, atuou também na concepção e coordenação do artigo.
O editorial de maio de 2020 da revista britânica Lancet aponta tais posturas do governo brasileiro como um dos grandes problemas para o enfrentamento da pandemia no país ${ }^{21}$.

A pandemia nos faz pensar sobre a produção de sentidos sobre cuidados por homens e mulheres e as condições e possibilidades que nos são ofertadas ou reguladas, na ordem social desigual que se mantém na sociedade. Para pensar estratégias de contenção da pandemia, é preciso ter atenção à relação entre gênero e cuidado. Faz-se também reconhecer a diversidade de posições de sujeito assumida por homens, considerando inclusive um olhar para as populações de homens historicamente estigmatizadas e muitas vezes alijadas do direito a usufruir de adequadas políticas públicas de saúde, como a população LGBTQI+, negros, indígenas, quilombolas, ribeirinhos, entre outras.

Noções de invulnerabilidade masculina, de hipervalorização da virilidade e de abjeção masculina ao cuidado e à prevenção são repertórios recorrentes (embora não recentes) que reificam o modelo central de uma ordem masculina que precisa se tornar objeto de reflexão, na medida em que colocam em risco à saúde de homens e mulheres e mais amplamente dos pactos civilizatórios e da ordem social. 


\section{Referências}

1. World Health Organization (WHO). Coronavirus Disease (COVID-19) Dashboard. [acessado 2020 Jun 24]. Disponível em: https://covid19.who.int/

2. Chen N, Zhou M, Dong X, Qu J, Gong F, Yang Han, Qiu Y, Wang J, Liu Y, Wei Y, Xia J, Yu T, Zhang Z, Zhang L. Epidemiological and clinical characteristics of 99 cases of 2019 novel coronavirus pneumonia in Wuhan, China: a descriptive study. Lancet 2020; 395:507-513. [acessado 2020 Jun 25]. Disponível em: https://www.thelancet.com/journals/lancet/article/ PIIS0140-6736(20)30211-7/fulltext

3. Jaillon S, Berthenet K, Garlanda C. Sexual dimorphism in innate immunity. Clin Rev Allergy Immunol 2019; 56(3):308-321.

4. Lyra J, Medrado B, Nascimento MAF, Alvarenga EC, Azevedo M, Correa ACP, Beiras A, Lima MLC. Implementação da Política Nacional de Atenção Integral à Saúde do Homem Reflexões Metodológicas. In: X Simpósio Brasileiro de Psicologia Política, 2018, Maceió/AL. Anais do X Simpósio Brasileiro de Psicologia Política. Belo Horizonte/MG: Associação Brasileira de Psicologia Política (ABPP), 2018. p. 116-116.

5. Baker P. From the Margins to the Mainstream: Advocating the Inclusion of Men's Health in Policy. A scoping study. London: Global Action on Men's Health; 2020.

6. Heise L, Greene ME, Opper N, Stavropoulou M, Harper C, Nascimento M, Zewdie D. Gender inequality and restrictive gender norms: framing the challenges to health. Lancet 2019; 393(10189):2440-2454.

7. Wenham C, Smith J, Morgan. COVID-19: the gendered impacts of the outbreak. Lancet 2020; 395(10227):846-848.

8. Marques ES, Moraes CL, Hasselmann MH, Deslandes SF, Reichenheim ME. A violência contra mulheres, crianças e adolescentes em tempos de pandemia pela COVID-19: panorama, motivações e formas de enfrentamento. Cad Saude Publica 2020; 36:e00074420.

9. Leal OF, Boff AM. Insultos, queixas, sedução e sexualidade: fragmentos de identidade masculina em uma perspectiva relacional. In: Parker R, Barbosa R, organizadores. Sexualidades Brasileiras. Rio de Janeiro: Relume-Dumará; 1996. p. 119-135.

10. Brasil. Número de pedidos de seguro-desemprego aumentou 1,3\% em 2020. [acessado 2020 Maio 13]. Disponível em: https://www.gov.br/economia/pt-br/ assuntos/noticias/2020/maio/numero-de-pedidosde-seguro-desemprego-aumentou-1-3-em-2020

11. El País Brasil. Desemprego nos EUA chega a 14,7\%, o mais alto em 70 anos. [acessado 2020 Maio 13]. [acessado 2020 Maio 13]. Disponível em: https://brasil. elpais.com/economia/2020-05-08/desemprego-nos -eua-chega-a-147-o-mais-alto-em-70-anos.html

12. Arilha M, Unbehaum S, Medrado B. Homens e masculinidades: outras palavras. São Paulo: Editora 34; 1998.
13. Almeida MV. Gênero, Masculinidade e Poder: Revendo um caso do sul de Portugal. In: Anuário Antropológico, 95. Rio de Janeiro: Tempo Brasileiro; 1996. p. 161-190.

14. Organización Panamericana de la Salud (OPS). Masculinidades y salud en la región de las Américas. Washington: OPS; 2019.

15. Baker P, White A, Morgan R. Men's health: COVID-19 pandemic highlights need for overdue policy action. Lancet 2020; 395(10241):1886-1888.

16. Medrado B, Lyra J. Por uma matriz feminista de gênero para os estudos sobre homens e masculinidades. Revista Estudos Feministas 2008; 16:809-840.

17. Almeida MV. Senhores de si. Uma interpretação antropológica da masculinidade. Lisboa: Fim de Século; 1995.

18. JGF. Algunas reflexiones sobre dilemas éticos del cuidado en un entorno de pandemia. Psicologia o Sociedade 2020; 32:e020003. [acessado 2020 Set 4]. Disponível em: https://www.scielo.br/scielo.php?script $=$ sci_arttext\&pid=S0102-71822020000100402\& $\operatorname{lng}=\mathrm{pt} \& \mathrm{nrm}=\mathrm{iso} \& \operatorname{lng}=\mathrm{es}$

19. UOL. Bolsonaro diz que é preciso 'enfrentar vírus como homem e não como moleque'. [acessado 2020 Maio 13]. Disponível em: https://noticias.uol.com.br/ultimasnoticias/agencia-estado/2020/03/29/bolsonaro-dizque-e-preciso-enfrentar-virus-como-homem-e-naocomo-moleque.htm

20. UOL. Diferente do discurso de Bolsonaro, "histórico de atleta" não salva competidores profissionais do coronavírus. [acessado 2020 Maio 13]. Disponível em: https://jc.ne10.uol.com.br/colunas/vida-fit/2020/03/ 5603546--historico-de-atleta--nao-salva-competidores-profissionais-do-coronavirus.html

21. Prado B. COVID-19 in Brazil: "So what?". Lancet 2020; 395(10235):1461.

Artigo apresentado em 27/06/2020

Aprovado em 27/10/2020

Versão final apresentada em 29/10/2020

Editores chefes: Romeu Gomes, Antônio Augusto Moura da Silva 
